\title{
Der Coaching-Markt
}

\author{
Peter-Paul Gross • Michael Stephan
}

Online publiziert: 17. Juli 2015

(C) Die Autor(en) 2015. Dieser Artikel ist auf Springerlink.com mit Open Access verfügbar.

Zusammenfassung Im vorliegenden Beitrag werden Strukturdaten zum deutschen Coaching-Markt präsentiert, anhand von Tabellen und Abbildungen aus den Marburger Coaching-Studien wird die Popularität und den Verbreitungsgrad von Coaching-Dienstleistungen im deutschen Sprachraum belegt. Der vorliegende Beitrag beantwortet die folgenden Fragen: Wie entwickelt sich der deutsche Coaching-Markt? Wie wird Coaching in Organisationen eingesetzt? Welche Umsätze werden durch Coaching erwirtschaftet?

Schlüsselwörter Marktentwicklung · Coaching-Markt · Marburger Coaching-Studien · Marktstrukturdaten · Organisation von Coaching

\section{The Coaching Market}

\begin{abstract}
This paper presents structural data of the German coaching market. Based on findings from the Marburger Coaching Surveys, the popularity and diffusion of coaching services within the German-speaking market are illustrated via tables and images. The paper answers the following questions: How is the German coaching market developing? How is coaching used in organizations? What are the turn overs generated by coaching services?
\end{abstract}

P.-P. Gross $(\bowtie)$

Metro AG,

Düsseldorf, Deutschland

E-Mail: peterpaulgross@gmail.com

M. Stephan

Wirtschaftswissenschaften, Philipps-Universität Marburg,

Marburg, Deutschland
Keywords Market development - Coaching market · Marburger coaching surveys - Structural data · Organization of coaching

\section{Einleitung}

Unter dem Stichwort Coaching finden sich bei „Google.de“ aktuell ungefähr 75 Mio. Einträge. „Google Scholar“, die Google-Suche für wissenschaftliche Texte, listet zum Suchbegriff Coaching etwa 700.000 Ergebnisse. Recherchiert man beim Online-Handelshaus Amazon.de nach Coaching werden fast 21.000 Treffer angezeigt, darunter fast 5000 deutschsprachige Bücher und über 14.500 fremdsprachige Bücher zum Thema. Diese Zahlen machen eines klar: Coaching ist populär! Diese Popularität bezieht sich dabei offensichtlich nicht nur auf Angebote von Coaching-Dienstleistungen, sondern auch auf Leistungen und Literatur rund um und über Coaching.

Trotz des offensichtlichen Erfolges der Dienstleistung Coaching ist das Verständnis dessen, was man eigentlich unter diesem Begriff zu verstehen hat, sehr heterogen. Dies liegt nicht zuletzt daran, dass auf Grund einer steigenden Nachfrage im Weiterbildungsmarkt nach Coaching, viele Weiterbildungsanbieter ihre Angebote durch das Anhängsel ,Coaching' zu adeln versuchen (Rauen 2008). In diesem Zusammenhang verwundert es nicht, dass die Vermutung des ,alten Weins in neuen Schläuchen“ immer wieder Teil der Diskussion ist (Taffertshofer 2007). Durch das uneinheitliche Begriffsverständnis und den nicht vorhandenen Schutz der Berufsbezeichnung, Coach` besteht jedoch die Gefahr, dass Coaching zu einem Container-Begriff verkommt und damit langfristig an Seriosität und Erfolg verliert.

Um sich mit Coaching aus wissenschaftlicher Sicht beschäftigen zu können, ist es daher wichtig, den Begriff 
erst einmal zu klären und damit von anderen Angeboten abzugrenzen. Aus ökonomischer Sicht handelt sich beim Coaching um eine besondere Form einer personenbezogenen, wissensintensiven Beratungsdienstleistung (Stephan et al. 2010). Umfassendere inhaltsbezogene Coaching-Definitionen finden sich bspw. bei Fengler (2001), Rauen (2005 und 2008a) oder Greif (2008). Bei einem Coaching arbeitet i.d. R ein Dienstleister (Coach) mit einem Klienten/Kunden (Coachee) im Umfang von meist 3-10 Sitzungen an einem zuvor vereinbarten Anliegen. Im organisationalen Kontext können Kunde und Klient zwei verschiedene Parteien sein, dann nämlich, wenn die Rahmenbedingungen der Coaching-Dienstleistung i.w.S. von einem Unternehmen (i.d. R vertreten durch einen Personalentwickler - auch Gatekeeper genannt) eingekauft und verhandelt werden. Das Anliegen i.e.S. wird jedoch meist in Einzelabsprache zwischen Coach und Coachee verhandelt und bearbeitet. Damit stellen Coaching-Prozesse i.e.S. aus organisationaler Sicht eine Art „Black Box“ dar, deren Inhalte nicht aktiv und ex nunc kontrolliert und gesteuert werden können.

Mit Blick auf Zielgruppen und Märkte lassen sich Coaching-Angebote nach ihrem Anlass in zwei Gruppen aufteilen. Dabei handelt es sich einerseits um sog. Life-Coaching-Angebote. Unter Life-Coaching fallen alle Angebote, die sich auf primär private Anliegen und Anlässe beziehen. Im Coaching-Segment der privaten Anliegen finden sich häufig auch eher „schillernd“ anmutende Coaching-Anlässe - Angebote wie Koch-Coaching, Astro-Coaching, GlücksCoaching bis hin zu Sex-Coaching zeugen hiervon. LifeCoaching-Angebote lassen sich andererseits abgrenzen von Business-Coaching. Beim Business-Coaching stehen primär berufsbezogene Anliegen und Anlässe im Fokus des Coachings, also bspw. Karriere, Leadership oder Transformationsthemen. Natürlich kann es auch im Business-Coaching vorkommen, dass private Themen zum Inhalt des Coachings werden. Grundsätzlich ist das formale Ziel eines Coachings im beruflichen Kontext i.d. R jedoch der Erhalt oder die Steigerung der berufsbezogenen Motivations- und Leistungsfähigkeit eines Coachee und private Themen sollten einen Bezug zu diesen Zielen haben.

Neben Angeboten von Einzelcoaching im klassischen Vieraugenformat (z. B. Looss 1997) wurden in den letzten Jahren eine ganze Reihe weiterer Formate im BusinessCoaching entwickelt. Zu erwähnen sind das Team-Coaching (z. B. Schmid 2005), Gruppen-Coaching (z. B. Rückle 2005) und Organisationscoaching (z. B. Höher 2007). Aktuellere Entwicklungen sind die Führungskraft als Coach (z. B. Neges und Neges 2008) und Coaching mit neuen Medien (Geißler 2008; Geißler und Stephan 2014). Wenn im Folgenden über Coaching geschrieben wird, ist Business-Coaching gemeint. Bezüge zu ökonomischer Theorie unterstreichen den wirtschaftswissenschaftlichen Charakter dieses Beitrags.
Das Ziel dieses Beitrages ist es im Folgenden, Strukturdaten zum deutschen Coaching-Markt zu präsentieren, welche im Rahmen der Marburger Coaching-Studien in den letzten Jahren erhoben wurden. Anhand von Tabellen und Abbildungen aus den Marburger Coaching-Studien wird die Popularität und den Verbreitungsgrad von CoachingDienstleistungen im deutschen Sprachraum belegt.

\section{Methodisches Vorgehen der Marburger Coaching- Studien}

Forschungsdesign und Untersuchungsgang der Studien

Über die Strukturen und Funktionsweisen des CoachingMarktes ist bislang nur wenig bekannt. Das Ziel der Marburger Coaching-Studien ist die Schaffung größerer Transparenz auf dem sehr unübersichtlichen deutschen Coaching-Markt. Bislang wurden drei Markt-Studien für die Jahre 2009, 2011 und 2013 in einem Panel-Design durchgeführt. Da Coaching aus ökonomischer Sicht ein bislang noch recht wenig erforschtes Gebiet darstellt, wurde für die Datenerhebungen der drei Marburger Coaching-Studien ein ,Mixed-Methods'-Ansatz verwendet (Kelle 2008; Kühl et al. 2005). Dieses Forschungsdesign hat sich bei explorativen Zielsetzungen bewährt. Der Gang der Untersuchung erfolgte dabei in drei Schritten: a.) Qualitative Delphi-Befragung zur Exploration und Konkretisierung neuer Themenfelder für die Hauptuntersuchung (Online-Befragung); b.) Pretest des Online-Fragebogens zur Validierung des Fragebogendesigns; c.) Quantitative angelegte Online-Befragung (Hauptuntersuchung).

Der quantitativen Datenerhebung vorgeschaltet waren explorative teilstandardisierte Experteninterviews mit jeweils zehn Coachs und zehn Personalmanagern bzw. Entscheidern in Unternehmen, welche Coaching einsetzen. Diese Interviews hatten zum Ziel, im Zuge einer qualitativen Delphi-Befragung zur Ideenaggregation, relevante Themenfelder und Trends aus Sicht von Coachs und deren Kunden einzugrenzen. Nachdem in einer ersten Interviewrunde relevante Themenfelder eingegrenzt werden konnten, wurden diese Themenfelder in einer zweiten Interviewrunde konkretisiert und Fragestellungen für die quantitative Überprüfung entwickelt. Zur Validierung des Fragebogendesigns wurde bei jeder der drei Marburger Coaching-Studien ein Pretest vorgeschaltet. Die quantitative Erhebung erfolgte mit getrennten Fragebögen für die Angebots- und Nachfrageseite des Marktes.

Am Pretest der Fragebögen für die 3. Marburger Coaching-Studie 2013 beteiligten sich für die Teilerhebung der Angebotsseite zwölf Coachs, auf der Nachfrageseite partizipierten zehn Unternehmen bzw. Personalmanager. Die quantitative Datenerhebung erfolge für die 3. Studie im 
Abb. 1 Stichprobenumfang und Teilnehmer der Marburger Coaching Studien

\begin{tabular}{|c|c|c|c|c|c|}
\hline & \multicolumn{2}{|c|}{ Fragebogenerhebung } & \multicolumn{2}{|l|}{ Pretest } & \multirow[b]{2}{*}{ Erhebungszeitraum } \\
\hline & Unternehmen & Coachs & Unternehmen & Coachs & \\
\hline Studie 2013 & 133 & 971 & 10 & 12 & $02 / 2013-05 / 2013$ \\
\hline Studie 2011 & 237 & 1282 & 17 & 15 & 05/2011-09/2011 \\
\hline Studie 2009 & 243 & 1090 & 9 & 12 & $12 / 2008-03 / 2009$ \\
\hline
\end{tabular}

Zeitraum Februar 2013 bis Mai 2013 mit Hilfe von OnlineFragebögen separat für die Anbieter (Coachs) und Nachfrager (Unternehmen) im deutschsprachigen Coaching-Markt. Für beide Marktseiten umfasste der Fragebogen wie auch in den Vorgängerstudien zwei Teile: Neben wiederkehrenden Strukturdateninformationen im ersten Teil, gab es jeweils auch einen thematischen Schwerpunkt der Studien. Der thematische Schwerpunkt in der Studie 2013 wurde auf den Einsatz von neuen Medien im Coaching gelegt. Insgesamt nahmen 971 Coachs auf der Angebotsseite und 133 Unternehmen für die Nachfrageseite des Marktes teil. Die nachfolgende Abbildung gibt einen zusammenfassenden Überblick über die Teilnehmerzahlen an den drei Marburger Coaching-Studien. Den Stichproben der drei Coaching-Studien kann ein „beinahe“ Panelcharakter attestiert werden, da sowohl auf der Angebots- als auch auf der Nachfrageseite ein Großteil der Befragten an allen drei Studien teilgenommen hat (Abb. 1).

\section{Wahl der Stichprobe}

Die Marburger Coaching-Studien adressieren Business Coaching, d. h. sie fokussieren sich auf Coachs, die ihre Leistungen im professionellen Kontext, also für Fach- und Führungskräfte in Unternehmen oder in nicht erwerbswirtschaftlich tätigen Organisationen (Non-Profit-Organisationen) anbieten. In allen Marburger Coaching-Studien wurden die Studienteilnehmer auf der Angebotsseite deshalb gebeten, sich nur an der Umfrage zu beteiligen, wenn sie Coaching zu primär beruflichen Anliegen und Anlässen (i.e.S. also Business-Coaching) anbieten.

Die Erhebungen der Marburger Coaching-Studien basieren auf einer offenen Grundgesamtheit, d. h. weder bei der Zielgruppe der Business-Coachs noch bei der Zielgruppe der Unternehmen, welche Coaching tatsächlich nutzen, ist die Grundgesamtheit präzise zu ermitteln. Da sich sowohl auf der Angebots- als auch auf der Nachfrageseite die Grundgesamtheit nicht mit Bestimmtheit ermitteln lässt, wurde mit dem Ziel maximaler Repräsentativität ein möglichst großer Rücklauf angestrebt. Zu diesem Zweck wurden sämtliche professionellen Coach-Datenbanken, -Plattformen und -Kontaktforen sowie Coaching-Verbände im deutschsprachigen Raum für die Ansprache genutzt. Darüber hinaus konnten 3355 Coachs direkt kontaktiert werden. Die Kontaktdaten stammen von den Teilnehmern der ersten beiden Marburger Coaching-Studien, welche ihre Emailadressen für die Ergebniszusendung angegeben hatten sowie einer ergänzenden Print-Medien-Analyse. Nach Abschluss des Erhebungszeitraums der 3. Studie lagen auf der CoachingAngebotsseite $N=971$ Datensätze vor. Das entspricht einem Anteil von etwa $12 \%$ der geschätzten Grundgesamtheit von 8000 Business-Coachs. Die Einschätzung der Grundgesamtheit von 8000 Business-Coachs basiert auf einer Einschätzung von 2009, in welcher die Einkommenssteuerdaten von Coachs zu Rate gezogen wurden (Stephan et al. 2010) sowie einer Bereinigung durch die Entwicklung des Marktwachstums der letzten Jahre (siehe dazu Marburger Coaching-Studien).

Bei der Stichprobenauswahl seitens der Unternehmen wurde darauf Wert gelegt, dass die Studie einen möglichst repräsentativen Überblick über all jene unternehmerischen Akteure gibt, welche sich bewusst mit Personalentwicklungsmaßnahmen beschäftigen und bereits Erfahrung beim Einsatz von Coaching gesammelt haben. Als Ansprechpartner in den betreffenden Unternehmen wurden diejenigen Personen befragt, die für das Management und die Organisation der Coaching-Maßnahmen verantwortlich sind (sog. Gatekeeper) im weiteren Kunden genannt. Die Gesamtpopulation der Kunden wurde somit auf jene Teilpopulation eingegrenzt, welche sich gezielt mit dem Thema Personalentwicklung und Coaching beschäftigt. Ähnlich wie bei der Auswahl der Coachs wurde versucht, innerhalb der Zielgruppe eine möglichst breite und zufallsgesteuerte Auswahl der Stichprobenelemente zu treffen, indem verschiedene Verbände sowie Personalmanager-Datenbanken, -Plattformen und spezialisierte Kontaktforen für die Ansprache genutzt wurden. Über eine Print-Medien-Analyse, die Kontaktdaten der Teilnehmer aus den Vorgängerstudien sowie über das Business-Netzwerk Xing konnten 718 Kunden direkt angeschrieben werden. Nach Abschluss der Erhebung lagen $N=133$ Datensätze für die Nachfrageseite vor. Für den exakten Zuschnitt der Stichprobe auf die Zielgruppe derjenigen Kunden mit Coaching-Erfahrung und damit zugunsten der Aussagekraft der Ergebnisse wurde eine geringere Stichprobengröße in Kauf genommen.

Im Folgenden werden ausgewählte Ergebnisse der Marburger Coaching-Studien präsentiert, welche die Popularität und Verbreitung von Coaching im deutschen Sprachraum belegen. Nicht alle Studienteilnehmer/innen machten vollständige Angaben in den Onlinefragebögen. Dieser Umstand wurde bei den entsprechenden Antworten durch Angaben zum Stichprobenanteil ( $n$ ) Rechnung getragen. 
Abb. 2 Zeitpunkte seit denen Kunden Coaching nachfragen und Coachs in den Markt eintreten
Entwicklung von Angebot und Nachfrage nach Coaching

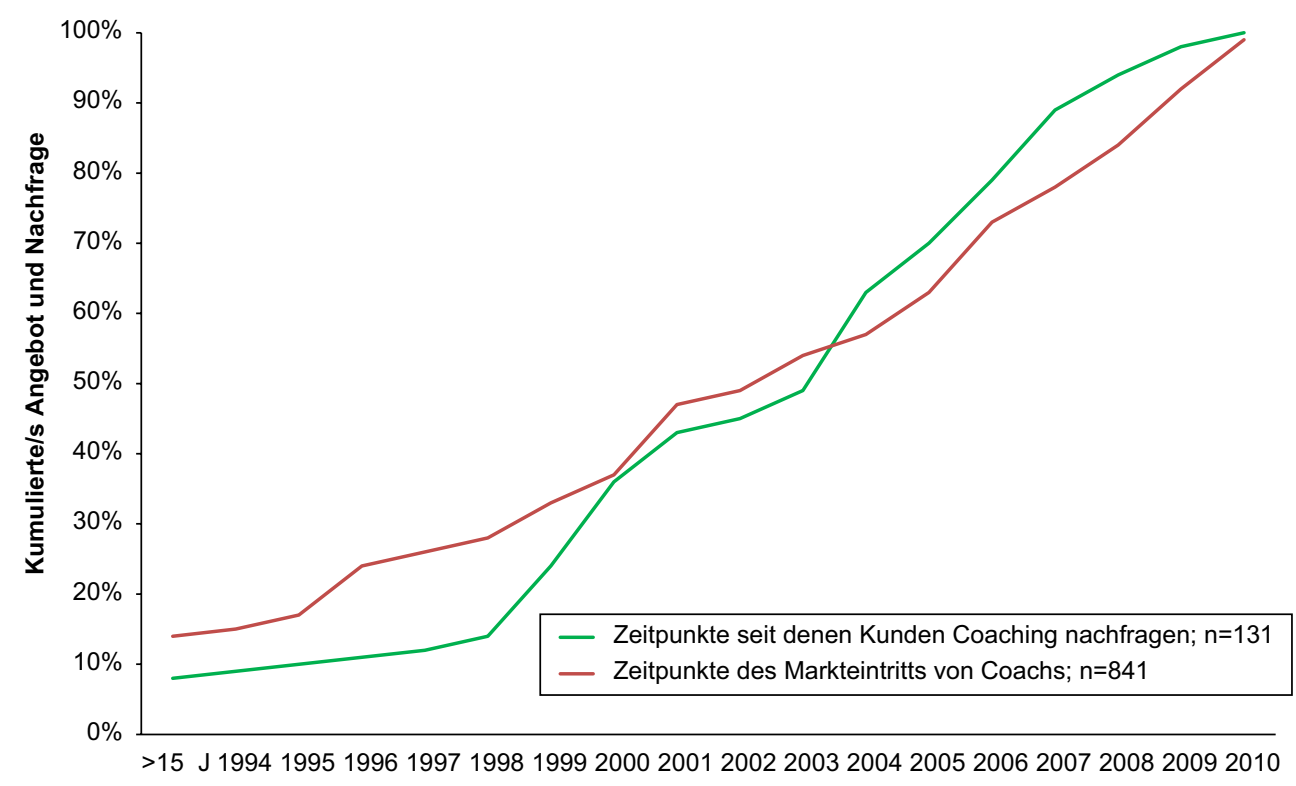

Um den Informationsgehalt der Ergebnisse möglichst hoch zu halten, ist es in den Wirtschafts- und Sozialwissenschaften üblich, zur deskriptiven Darstellung auch unvollständige Fragebögen in die Auswertung mit einzubeziehen. Angaben die im Folgenden als „Durchschnitt“ oder ,durchschnittlich" bezeichnet werden, beziehen sich, wenn nicht anders benannt, immer auf den arithmetischen Mittelwert.

\section{Strukturelle Entwicklung des Coaching-Marktes}

Aus der Sportpsychologie kommend, wurde Coaching zum Ende der 1980er, Anfang der 1990er Jahre als Beratungsmaßnahme für Top-Führungskräfte sowohl in Deutschland wie auch den USA eingeführt. Ziel war es, die zwischenmenschlichen und insbesondere die führungsbezogenen Kompetenzen im Top-Management zu verbessern (Hogan et al. 1994; Charan und Colvin 1999; Dotlich und Cairo 1999). Um Führungsqualitäten zu entwickeln, Beförderungen vorzubereiten oder mit dem organisationalen Wandel besser zu Recht zu kommen, begannen Unternehmen für ihre Führungskräfte (und teilweise auch die Führungskräfte selbst) Beraterverträge mit externen Coachs abzuschließen (Greco 2001; Niemes 2002). Diesen Trend seit Beginn der 1990er Jahre belegt Abb. 2. Die Abbildung visualisiert in kumulierter Form die Zeitpunkte, zu denen die in der Marburger Coaching-Studie befragten Kunden zum ersten Mal von Coaching gehört haben und Markteintrittszeitpunkte der befragten Coachs.

Die Gründe für die gestiegene Popularität von Coaching lassen sich auf zwei Ebenen verorten: 1) Einerseits haben sich die extremen Rahmenbedingungen vieler Branchen und Märkte verändert, in denen Unternehmen und ihre Mitarbeiter agieren. Durch die Internationalisierung des Wettbewerbs, durch schnellere Produkt- und Innovationszyklen, die Verbreitung von Informations- und Kommunikationstechnologien sowie dem permanent geforderten organisatorischen Wandel sind auch die Anforderungen an die Fach-und Führungskräfte hinsichtlich der Komplexität ihrer Aufgaben, der Flexibilität, Zielorientierung, Kommunikationsfähigkeit, sozialen Kompetenzen etc. gestiegen. 2) Andererseits hat sich auch die Führungs-und Personalkultur in den Unternehmen gewandelt: Die Mitarbeiterzufriedenheit ist in die obersten Ebenen der Zielhierarchien vorgerückt. Unternehmen haben erkannt, dass ihre Mitarbeiter die wertvollste Ressource darstellen, die es zu pflegen gilt. Der Druck zur permanenten organisatorischen Wandlungsfähigkeit fordert die Mitarbeiter und insbesondere die Führungskräfte. Zur Bewältigung dieser Herausforderung setzen Unternehmen neben Instrumenten, die an strategische, organisatorische und kulturelle Bedingungen anknüpfen, auf personenbezogene Maßnahmen des Change Managements: Mit Hilfe von Personalentwicklungsmaßnahmen, insbesondere Coaching, versuchen sie, das Potenzial ihrer Mitarbeiter $\mathrm{zu}$ aktivieren und $\mathrm{zu}$ erschließen, um deren Lernbereitschaft und Lernfähigkeit als Grundlage für ihre Veränderungsfähigkeit zu erhöhen.

Coaching hat sich in den vergangenen 20 Jahren zum populärsten Format unter den Personalentwicklungsinstrumenten im Management entwickelt. Der Umsatz im deutschen Coaching-Markt betrug in 2012, nach konservativen Berechnungen, ca. 350 Mio. $€$. Von den 350 Mio. $€$ entfallen allein ca. 20 Mio. $€$ auf den Markt für Coaching-Weiterbildung, der durch neu in den Markt eintretende Coachs und 
Abb. 3 Unternehmensgrößen von Coaching-Kunden

\begin{tabular}{|l|r|r|r|r|r|}
\hline Wie oft arbeiten Sie für die folgenden Kundengruppen? \\
\hline $\mathrm{n}=728$ & se hr oft & \multicolumn{1}{|c|}{ oft } & gelegentlich & \multicolumn{1}{c|}{ selten } & nie \\
\hline Einzelpersonen unternehmensunabhängig & $29,4 \%$ & $24,5 \%$ & $28,0 \%$ & $13,3 \%$ & $4,8 \%$ \\
\hline kleine Unternehmen bis 10 Mitarbeiter & $7,1 \%$ & $17,7 \%$ & $26,1 \%$ & $28,5 \%$ & $20,7 \%$ \\
\hline kleine Unternehmen bis 50 Mitarbeiter & $4,8 \%$ & $17,1 \%$ & $32,5 \%$ & $25,1 \%$ & $20,5 \%$ \\
\hline kleine Unternehmen bis 100 Mitarbeiter & $3,3 \%$ & $17,7 \%$ & $34,1 \%$ & $22,7 \%$ & $22,3 \%$ \\
\hline mittlegroße Unternehmen bis 200 Mitarbeiter & $4,8 \%$ & $18,9 \%$ & $35,1 \%$ & $22,4 \%$ & $18,8 \%$ \\
\hline mittlegroße Unternehmen bis 500 Mitarbeiter & $5,1 \%$ & $25,3 \%$ & $33,7 \%$ & $18,1 \%$ & $17,8 \%$ \\
\hline mittlegroße Unternehmen bis 1.000 Mitarbeiter & $4,9 \%$ & $24,6 \%$ & $29,6 \%$ & $18,9 \%$ & $22,0 \%$ \\
\hline mittlegroße Unternehmen bis 5.000 Mitarbeiter & $8,4 \%$ & $24,2 \%$ & $27,3 \%$ & $17,6 \%$ & $22,5 \%$ \\
\hline große Unternehmen bis 10.000 Mitarbeiter & $8,4 \%$ & $19,9 \%$ & $20,5 \%$ & $16,2 \%$ & $35,0 \%$ \\
\hline große Unternehmen bis 25.000 Mitarbeiter & $6,2 \%$ & $16,2 \%$ & $17,4 \%$ & $16,8 \%$ & $43,4 \%$ \\
\hline große Unternehmen bis 50.000 Mitarbeiter & $6,4 \%$ & $16,2 \%$ & $13,5 \%$ & $17,8 \%$ & $46,1 \%$ \\
\hline große Unternehmen bis 500.000 Mitarbeiter & $7,7 \%$ & $10,4 \%$ & $12,7 \%$ & $15,0 \%$ & $54,2 \%$ \\
\hline sehr große Unternehmen bis 600.000 Mitarbeiter & $5,3 \%$ & $5,5 \%$ & $8,4 \%$ & $15,6 \%$ & $65,3 \%$ \\
\hline
\end{tabular}

deren Ausbildungsinvestitionen sowie Weiterbildungsinvestitionen aktiver Coachs besteht. Das Marktvolumen von 330 Mio. $€$ für reine Coaching-Dienstleistungen berechnet sich über die Zahl der aktiven Coachs, deren Jahresumsätze multipliziert mit der geschätzten Grundgesamtheit von 8000 Business-Coachs. Über die letzten fünf Jahre verzeichnete der Markt ein durchschnittliches Wachstum p. a. von $40 \%$ und mehr. Dieses Wachstum ergibt sich aus den geschätzten steigenden Zahlen der Anbieter und deren Umsätzen in den letzten Jahren. Darüber hinaus ist das Wachstum nicht zuletzt dem Umstand geschuldet, dass immer mehr Dienstleistungen als Coaching deklariert und abgerechnet werden. Gemäß einer Studie des Harvard Business Managers (2010) zählen die Konzeption von Coaching-Maßnahmen und die Auswahl von Coachs mittlerweile zu den wichtigsten Aufgaben von Personalmanagern.

Wie entwickelt sich der Coaching-Markt aktuell? Diese Frage lässt sich aus verschiedenen Blickwinkeln beantworten. Ein wichtiger struktureller Aspekt auf der Nachfrageseite ist die Größe der Unternehmen, die Coaching einsetzen: Bislang galt Einsatz von Coaching als Domäne von Großunternehmen. Abbildung 3 gibt eine Übersicht über die Unternehmensgrößen der Kunden von CoachingDienstleistungen. Im Vergleich zu den Vorjahren arbeiteten deutlich weniger der befragten Coachs ,sehr oft“ für große Unternehmen. Dies mag an den Unterschieden in den Stich-
proben-Populationen im Vergleich zu den vorigen Studien liegen (lediglich 163 Coachs haben bereits in 2009 und 278 in 2011 an den Marburger Coaching-Studien teilgenommen), es kann sich aber auch um ein erstes Indiz für eine zunehmende Nachfrage bei kleinen und mittelständischen Unternehmen nach Coaching-Dienstleistungen handeln. Es bleibt abzuwarten, wie sich die Nachfrage in den kommenden Jahren entwickeln wird. Die Gründe für das private in Anspruch nehmen von Coaching-Dienstleistungen sind vielfältig und reichen von Testbuchern, über eine geringe Akzeptanz von Coaching beim Arbeitgeber bis hin zu sensiblen Coaching-Anlässen, von welchen der Arbeitgeber nichts erfahren soll.

Neben den Kundengruppen von Coachs stehen bei Abb. 4 die Branchen der Kunden von Coachs im Fokus. Im Vergleich mit den Angaben aus der zweiten Marburger Coaching-Studie von 2011 fällt auf, dass die Angaben zu Branchen, in denen Coachs arbeiten, seltener als ,sehr oft“ und „oft“ markiert wurden. Es ist fraglich, ob diese Angaben bereits als ein Indiz für eine nachlassende Nachfrage gesehen werden können. Festzuhalten ist, dass CoachingDienstleistungen nach wie vor in allen Branchen nachgefragt werden. Wie schon die Ergebnisse 2011 nahe legten, so lässt sich auch 2013 beobachten, dass ein verstärkter Einsatz von Coaching in Dienstleistungsbranchen (Kategorien 5-13) stattfindet. In der verarbeitenden Industrie scheint
Abb. 4 In welchen Branchen arbeiten Coachs?

\begin{tabular}{|c|c|c|c|c|c|}
\hline \multicolumn{6}{|c|}{ In welcher Branche arbeiten Sie? } \\
\hline$n=619$ & sehr oft & oft & gelegentlich & selten & nie \\
\hline Automobilindustrie & $6,0 \%$ & $9,7 \%$ & $30,5 \%$ & $19,2 \%$ & $34,6 \%$ \\
\hline Maschinenbau/Elektrotechnik & $6,6 \%$ & $16,4 \%$ & $28,2 \%$ & $19,1 \%$ & $29,6 \%$ \\
\hline Chemie/Pharma/Biotechnologie & $5,4 \%$ & $15,5 \%$ & $27,6 \%$ & $19,7 \%$ & $31,8 \%$ \\
\hline Sonstige verarbeitende Industrie & $3,2 \%$ & $18,4 \%$ & $31,5 \%$ & $23,2 \%$ & $23,8 \%$ \\
\hline Handel/Vertrieb & $8,9 \%$ & $22,3 \%$ & $31,0 \%$ & $20,3 \%$ & $17,5 \%$ \\
\hline Finanzen/Banken/Versicherungen & $12,2 \%$ & $18,0 \%$ & $28,4 \%$ & $18,2 \%$ & $23,3 \%$ \\
\hline IT/Software & $6,7 \%$ & $19,7 \%$ & $31,9 \%$ & $17,6 \%$ & $24,1 \%$ \\
\hline Medien/Komn & $7,8 \%$ & $16,8 \%$ & $25,4 \%$ & $22,5 \%$ & $27,5 \%$ \\
\hline Verwaltung/Öffentlicher D & $9,7 \%$ & $21,5 \%$ & $27,1 \%$ & $18,6 \%$ & $23,1 \%$ \\
\hline Bildung/Forschung & $5,9 \%$ & $19,6 \%$ & $29,5 \%$ & $20,1 \%$ & $24,8 \%$ \\
\hline Verkehr/Transport/Logistig/Reise & $3,6 \%$ & $7,7 \%$ & $20,5 \%$ & $24,8 \%$ & $43,4 \%$ \\
\hline Gesundheits-/Sozialwesen & $12,4 \%$ & $23,4 \%$ & $25,7 \%$ & $17,7 \%$ & $20,8 \%$ \\
\hline Sonstige Dienstleistungen & $12,5 \%$ & $26,2 \%$ & $34,2 \%$ & $15,6 \%$ & $11,5 \%$ \\
\hline
\end{tabular}


Abb. 5 Einsatzebenen von Coaching

Abb. 6 Coaching in anderen Sprachen

\begin{tabular}{|c|c|c|c|c|c|c|c|c|c|c|}
\hline \multicolumn{11}{|c|}{ Auf welchen Unternehmensebenen arbeiten Sie als Coach? } \\
\hline $\mathrm{n}=724$ (Coachs rot); $n=48$ (Kunden grün) & \multicolumn{2}{|c|}{ sehr oft } & \multicolumn{2}{|c|}{ oft } & \multicolumn{2}{|c|}{ gelegentlich } & \multicolumn{2}{|c|}{ selten } & \multicolumn{2}{|c|}{ nie } \\
\hline Top Management/Executives & $13,5 \%$ & 12, & $23,3 \%$ & & $27,0 \%$ & $29,2 \%$ & $20,7 \%$ & $12,5 \%$ & $15,5 \%$ & $8,3^{\circ}$ \\
\hline Gehobe & & & & & & 29 & & & $4,4 \%$ & 0, \\
\hline Mittler & 16 , & & & & & & 4,3 & & 2,8 & 2, \\
\hline chwuchsf & & 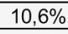 & 35 & & & & & & $6,3 \%$ & \\
\hline Mitarbeiter & $14,5 \%$ & $6,3 \%$ & $23,8 \%$ & $12,5 \%$ & $26,2 \%$ & $20,8 \%$ & $21,5 \%$ & $43,8 \%$ & $14,0 \%$ & $16,7 \%$ \\
\hline
\end{tabular}

\section{Coaching in anderen Sprachen}

\begin{tabular}{|l|r|r|r|r|r|r|r|r|r|r|}
\hline \multicolumn{2}{|c|}{$\mathrm{n}=343$ (Coachs rot); $\mathrm{n}=17$ (Kunden grün) } \\
\hline & \multicolumn{1}{|c|}{ sehr oft } & \multicolumn{2}{|c|}{ oft } & \multicolumn{1}{|c|}{ gelegentlich } & \multicolumn{2}{|c|}{ selten } & \multicolumn{2}{|c|}{ nie } \\
\hline Englisch & $14,0 \%$ & $35,3 \%$ & $14,9 \%$ & $35,3 \%$ & $56,0 \%$ & $17,6 \%$ & $13,1 \%$ & $11,8 \%$ & $2,0 \%$ & $0,0 \%$ \\
\hline Spanisch & $2,5 \%$ & $0,0 \%$ & $1,3 \%$ & $10,0 \%$ & $5,0 \%$ & $10,0 \%$ & $6,9 \%$ & $20,0 \%$ & $84,4 \%$ & $60,0 \%$ \\
\hline Französisch & $2,4 \%$ & $0,0 \%$ & $4,1 \%$ & $16,7 \%$ & $12,4 \%$ & $16,7 \%$ & $12,9 \%$ & $41,7 \%$ & $68,2 \%$ & $25,0 \%$ \\
\hline Russisch & $0,7 \%$ & $0,0 \%$ & $0,7 \%$ & $0,0 \%$ & $0,7 \%$ & $10,0 \%$ & $0,0 \%$ & $30,0 \%$ & $98,0 \%$ & $60,0 \%$ \\
\hline Türkisch & $0,0 \%$ & $0,0 \%$ & $1,3 \%$ & $0,0 \%$ & $0,0 \%$ & $20,0 \%$ & $2,0 \%$ & $10,0 \%$ & $96,7 \%$ & $70,0 \%$ \\
\hline Chinesisch & $0,7 \%$ & $10,0 \%$ & $0,0 \%$ & $0,0 \%$ & $0,0 \%$ & $20,0 \%$ & $0,7 \%$ & $20,0 \%$ & $98,7 \%$ & $50,0 \%$ \\
\hline Arabisch & $0,0 \%$ & $0,0 \%$ & $0,0 \%$ & $0,0 \%$ & $1,3 \%$ & $10,0 \%$ & $0,7 \%$ & $20,0 \%$ & $98,0 \%$ & $70,0 \%$ \\
\hline Portugisisch & $2,0 \%$ & $0,0 \%$ & $0,0 \%$ & $0,0 \%$ & $2,0 \%$ & $10,0 \%$ & $0,0 \%$ & $30,0 \%$ & $96,0 \%$ & $60,0 \%$ \\
\hline
\end{tabular}

Coaching etwas weniger häufig eingesetzt zu werden (Kategorien 1-4). Diese Einschätzung deckt sich allerdings nicht mit den Angaben der Kunden.

Der nachfolgenden Abb. 5 kann man entnehmen, auf welchen Hierarchieebenen Coaching heute eingesetzt wird. In der Abbildung sind die Angaben der Coachs (rot) und die Angaben der Kunden (grün) zu den Hierarchieebenen ihrer Coachees dargestellt. Auffällig ist, dass, wie bereits in den letzten Jahren, die Schwerpunkt-Zielgruppe von CoachingDienstleistungen bei Führungskräften nach wie vor im mittleren und gehobenen Management liegt. 40,3\% der Coachs gaben an, ,oft" mit Coachees aus dem gehobenen Management zu arbeiten. 56,3\% der Kunden markierten diese Zielgruppe ebenfalls mit „oft“. 49,4\% der Coachs markierten auch das mittlere Management als Coachees, mit welchen „oft“ zusammen gearbeitet wird. $46 \%$ der Kunden bestätigten diese Einschätzung ebenfalls mit ,oft“.

Das Thema „Coaching in anderen Sprachen“" wurde, nach der 2. Marburger-Coaching-Studie 2011, erneut aufgegriffen. In der vergangenen Studie schien sich der Trend abzuzeichnen, dass die Nachfrage in diesem Bereich steigt. Die nachfolgende Abb. 6 greift dieses Thema deshalb auf und gewährt einen Einblick zur Häufigkeit des Einsatzes von Coaching in anderen Sprachen. Auffällig ist, dass mit Ausnahme von Englisch kaum eine andere Sprache stark nachgefragt wird. Coaching in anderen Sprachen stellt im deutschsprachigen Raum folglich nach wie vor eine Nische dar. Zu den Kunden von Coaching in anderen Sprachen zählen in den meisten Fällen internationale Unternehmen, die Coaching für ihr internationales Personal buchen. In den Jahren 2011 und 2013 lag der Anteil fremdsprachiger Coachings nach Angabe von Kunden im Durchschnitt in beiden Jahren bei $7 \%$.

Wie haben sich die Auftragszahlen für Coachs in den letzten Jahren verändert? Abbildung 7 gibt einen Überblick zur Entwicklung der Auftragslage von Coachs über die letzten Jahre. Es zeigt sich, dass nach einer steigenden Nachfrage

\begin{tabular}{|c|c|c|}
\hline \multicolumn{3}{|c|}{ Coaching-Aufträge als Externer } \\
\hline Jahr & $\varnothing$ Aufträge & $\mathbf{n}$ \\
\hline $\mathbf{2 0 0 6}$ & 17,1 & 275 \\
\hline $\mathbf{2 0 0 7}$ & 17,1 & 316 \\
\hline $\mathbf{2 0 0 8}$ & 19,8 & 368 \\
\hline $\mathbf{2 0 0 9}$ & 21,3 & 415 \\
\hline $\mathbf{2 0 1 0}$ & 24,4 & 513 \\
\hline $\mathbf{2 0 1 1}$ & 18,6 & 497 \\
\hline $\mathbf{2 0 1 2}$ & 20,9 & 550 \\
\hline
\end{tabular}

Abb. 7 Coaching-Aufträge als Externer

\begin{tabular}{|c|c|c|c|c|}
\hline \multicolumn{6}{|c|}{ Brutto Coaching-Stundensätze } \\
\hline Jahr & $\boldsymbol{n}$ & $\mathbf{n}$ (Coachs) & $\boldsymbol{n}$ & n (Kunden) \\
\hline $\mathbf{2 0 0 8}$ & 139,95 EUR & 466 & 213,80 EUR & 22 \\
\hline $\mathbf{2 0 0 9}$ & 148,60 EUR & 479 & 219,09 EUR & 23 \\
\hline $\mathbf{2 0 1 0}$ & 161,99 EUR & 521 & 216,73 EUR & 27 \\
\hline $\mathbf{2 0 1 1}$ & 174,40 EUR & 555 & 249,72 EUR & 37 \\
\hline $\mathbf{2 0 1 2}$ & 181,99 EUR & 635 & 263,69 EUR & 43 \\
\hline
\end{tabular}

Abb. 8 Brutto Coaching-Stundensätze

über die letzten Jahre für das Jahr 2011 ein leichter Rückgang in der Nachfrage zu verzeichnen war. Im Jahr 2012 zog die Nachfrage auf im Schnitt 20,9 Aufträge je Coach im Jahr wieder an, befindet sich damit allerdings noch nicht wieder auf dem gleichen Niveau wie 2010. Diese Entwicklung spiegelt sich auch in Abb. 9 bei den anteiligen Umsatzzahlen von Coachs wieder.

Trotz leichten Einbrüchen bei der Nachfrage scheinen sich die Stundensätze im Coaching weiter positiv zu entwickeln. Abbildung 8 gibt einen Überblick zur Entwicklung der Stundensätze im Coaching-Markt. Im Jahr 2012 lag der Durchschnitt der Stundensätze, die Coachs angaben, bei brutto $181,99 €$. Der Unterschied zum durchschnittlichen Stundensatz von 263,69 € der Kunden ist u. a. dadurch zu 


\begin{tabular}{|c|c|c|}
\hline \multicolumn{3}{|c|}{ Brutto Jahresumsatz f. Coaching } \\
\hline Jahr & $\boldsymbol{\emptyset}$ & $\mathbf{n}$ \\
\hline $\mathbf{2 0 0 8}$ & $22.974,39$ EUR & 262 \\
\hline $\mathbf{2 0 0 9}$ & $25.132,65$ EUR & 264 \\
\hline $\mathbf{2 0 1 0}$ & $30.060,30$ EUR & 281 \\
\hline $\mathbf{2 0 1 1}$ & $35.857,97$ EUR & 296 \\
\hline $\mathbf{2 0 1 2}$ & $41.025,17$ EUR & 349 \\
\hline
\end{tabular}

Abb. 9 Brutto Jahresumsätze von Coachs

erklären, dass bei den Kunden ausschließlich Unternehmenskunden befragt wurden, die üblicherweise höhere Stundensätze ansetzten, während Privatkunden mit z. T. deutlich geringeren Stundensätzen auf der Nachfrageseite ausgeblendet bleiben.

Neben den Stundensätzen im Coaching werden Coachings häufig auch mit Tagessätzen berechnet. Der durchschnittliche Brutto-Tagessatz lag im Jahr 2012 bei 1259,41 €. Das absolute Maximum für einen Tagessatz lag bei $15.000 €$. Nach Angaben der Kunden lag das durchschnittliche Tages- honorar bei $1494,47 €$, wobei minimal $180 €$ und maximal $2500 €$ gezahlt wurden. Der durchschnittliche Jahresumsatz, den Coachs erwirtschafteten ist über die letzten Jahre trotz der in Abb. 7 vermuteten nachlassenden Nachfrage weiter auf durchschnittlich 41.025,17€ gestiegen (Abb. 9).

Wirft man einen Blick auf die Umsatzanteile, die Coachs in den letzten Jahren durch Coaching erwirtschaftet haben (Abb. 10), so fällt auf, dass es im Jahr 2011, korrespondierend mit den Angaben aus Abb. 7, einen „kleinen Einbruch“" gegeben hat. Der Anteil der Coachs, die angaben, zu 100\% von Coaching zu leben, sank auf $8 \%$ und verharrte auch 2012 auf diesem Wert. Auch der Anteil der Umsätze, den Coachs durchschnittlich allein durch Coaching erwirtschaften, sank 2011 auf 35\%. Dieser stieg jedoch 2012 bereits wieder auf $37 \%$ an.

\section{Strategien und Organisation der Nachfrage nach Coaching}

Verglichen mit anderen Personalentwicklungsdienstleistungen wie Training oder Führungskräfteprogramme ist der Einsatz von Coaching teuer und erzeugt damit aus organisa-

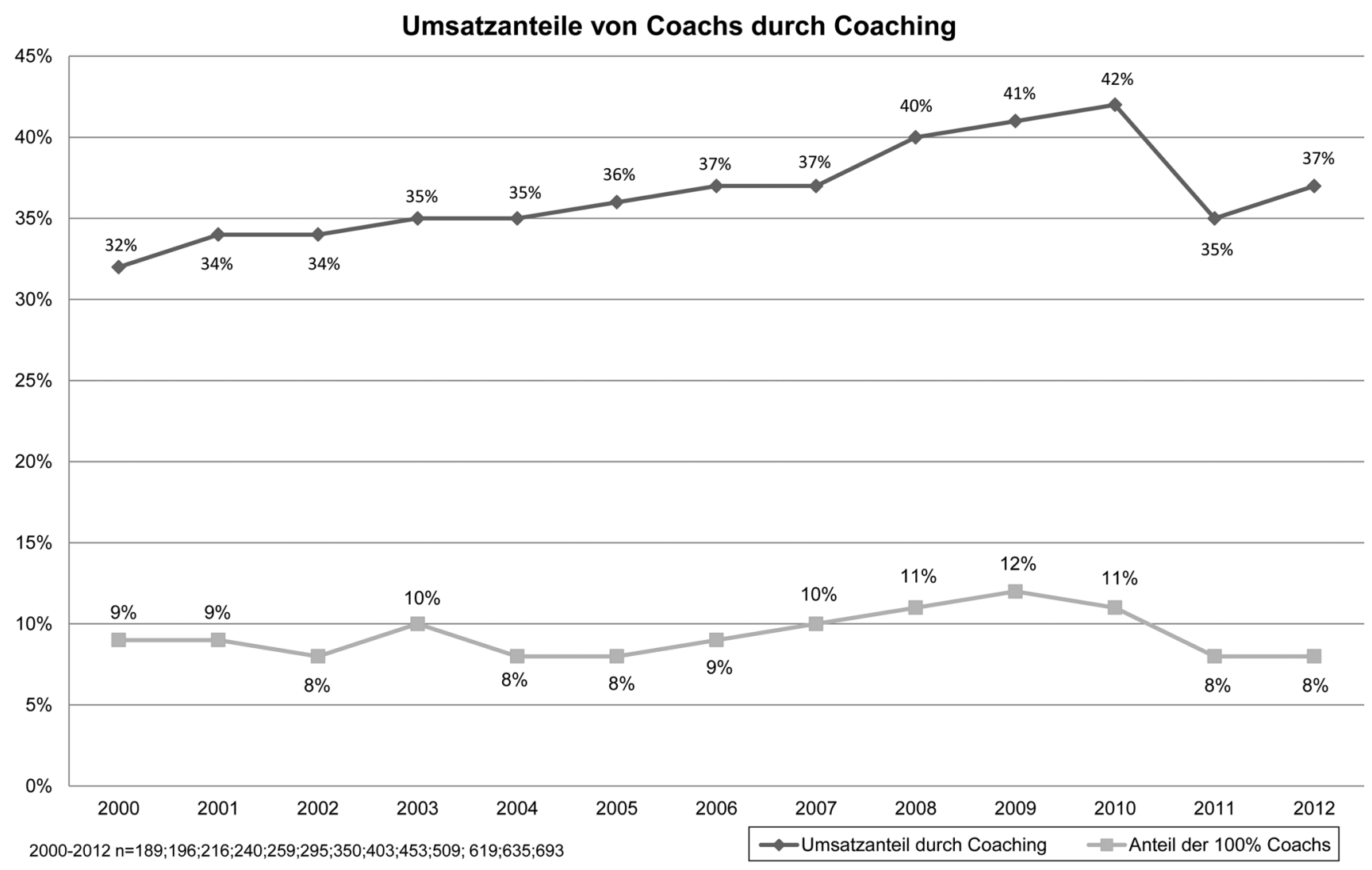

Abb. 10 Umsatzanteile von Coachs durch Coaching 
torischer Sicht pro Person höhere Kosten, welche sich leider nicht auf seriöse Weise einem quantifizierbaren Return on Investment (ROI) zurechnen lassen. Es ist also verständlich, dass die Nachfrager von Coaching-Dienstleistungen versuchen, die Kosten für den Einsatz von Coaching zu reduzieren. Betrachtet man einen strukturierten CoachingProzess, so lassen sich zwei kritische Kostentreiber identifizieren, die im Zuge der Organisation und der Abwicklung von Coaching-Maßnahmen entstehen (im ökonomischen Duktus: sogenannte „Transaktionskosten"). Gemeint sind hiermit insbesondere die Kosten der Suche und Auswahl eines passenden Dienstleisters sowie die Kosten, die dem Unternehmen entstehen, wenn man sich für den falschen Coach entscheidet. Beide Kostentreiber lassen sich durch den Einsatz eines sogenannten Coaching-Pools reduzieren.

Eine weitere Möglichkeit zur Kostenreduktion bietet der Einsatz von sog. internen Coachs. Dabei handelt es sich meist um Mitarbeiter der Organisation, die eine Coaching-Ausbildung absolviert haben und $\mathrm{zu}$ internen Verrechnungspreisen Coaching-Dienstleistungen innerhalb der Organisation anbieten.

Gerade für große Unternehmen, die einen hohen und regelmäßig auftretenden Coaching-Bedarf haben, der nicht (ausschließlich) über interne Anbieter gedeckt werden kann, ist der Unterhalt eines Coaching-Pools ein angemessener Weg, um neben der Kostenreduktion Coaching strategisch im Unternehmen zu verankern und die Qualität im Coaching zu sichern. Ein Coaching-Pool umfasst bei großen Unternehmen meist all jene Coachs, mit denen ein Rahmenvertrag geschlossen wurde. Ein von Unternehmen aufgebauter Coaching-Pool muss aber nicht notwendigerweise nur externe Anbieter umfassen. Auch interne Coachs können ergänzend dazu in den Pool aufgenommen werden. Ziel der Etablierung eines Coaching-Pools ist es jedoch in jedem Fall, ein dauerhaftes Netzwerk mit verschiedenen externen und/oder internen Coaching-Anbietern aufzubauen, die dann zielgruppen- und maßnahmenspezifisch eingesetzt werden können. Kern eines Coaching-Pools ist eine CoachDatenbank, die neben elementaren biografischen Daten und Informationen zur Qualifikation und Spezialisierung auch die konkreten Ergebnisse der durchgeführten CoachingMaßnahmen, Honorarspannen sowie Feedback der Coa- chees (Evaluationsergebnisse) enthalten. In der Regel wird die Coach-Datenbank im Intranet der Unternehmen den Mitarbeitern zugänglich gemacht. Die endgültige bzw. nachhaltige Aufnahme von Coachs in den Pool erfolgt aufgrund positiver Erfahrungen in der Vergangenheit oder auf Basis eines Audit-Verfahrens, dem sich die Coachs unterziehen müssen. Coaching-Pools lassen sich auch im Zusammenschluss von Unternehmen einsetzen. Gerade für kleine- und mittelständische Unternehmen, die keinen hohen und regelmäßig auftretenden Coaching-Bedarf haben, erscheint die Kooperation mit anderen Unternehmen in der Personalentwicklung sinnvoll. In den letzten Jahren ist es deshalb vermehrt zu beobachten, dass sich Unternehmen (nicht notwendigerweise aus denselben Branchen) zusammenschließen und gemeinschaftliche Coaching-Pools etablieren und administrieren (Gross und Stephan 2011). In manchen Wirtschaftszweigen werden diese Aktivitäten auch von den entsprechenden Branchenverbänden unterstützt (Gross und Stephan 2011). 53\% der befragten Kunden $(n=74)$ gab an einen Coaching-Pool im Unternehmen zu unterhalten.

Vor der Aufnahme in den unternehmensinternen Coaching-Pool stellt sich zunächst einmal die Frage, wie Kunden eigentlich auf externe Coachs aufmerksam werden. Dieser Frage wurde in Abb. 11 nachgegangen. Aufgelistet sind Kriterien, die bei der Identifikation von Coachs eine Rolle spielen. Es bestätigt sich die verbreitete Annahme, dass Coaching in der Anbahnung ein Beziehungsgeschäft zu sein scheint. Immerhin halten 74,6\% der Kunden Empfehlungen, Mund-zu-Mund-Kommunikation sowie Meinungen aus dem eigenen Netzwerk für ,sehr wichtig“ oder „wichtig“ (22,5\%). Ebenfalls „wichtig“ sind offenbar die Referenzen von Coachs aus dem eigenen Coaching-Pool. So markierten 38,3\% der Kunden Gespräche mit Coachs des eigenen Coaching-Pools über neue Coachs als „sehr wichtig“ oder ,wichtig“ (36,7\%).

Entscheidend ist nach der Sichtung jedoch, welche Kriterien denn nun tatsächlich eine Rolle bei der Auswahl von Coachs für den eigenen Coaching-Pool spielen. Die nachfolgende Abb. 12 bezieht sich genau auf diese Frage. Vierfach skaliert sind Auswahlkriterien durch Kunden gewichtet worden.
Abb. 11 Kriterien zur Identifikation von externen Coachs

\begin{tabular}{|l|c|c|c|c|}
\hline Kriterien von Kunden, die zur Identifikation von externen Coachs eine Rolle spielen \\
\hline $\mathrm{n}=71$ & sehr wichtig & wichtig & weniger wichtig & unwichtig \\
\hline Empfehlungen, Mund-zu-Mund, Meinungen aus meinem Netzwerk & $74,6 \%$ & $22,5 \%$ & $2,8 \%$ & $0,0 \%$ \\
\hline Netzwerkveranstaltungen z.B. Messen, Infostände, etc. & $7,2 \%$ & $33,3 \%$ & $37,7 \%$ & $21,7 \%$ \\
\hline Gezielte Suche im Internet z.B. google, Xng, etc. & $5,8 \%$ & $27,5 \%$ & $47,8 \%$ & $18,8 \%$ \\
\hline Gespräche mit Coachs des eigenen Coaching-Pools über neue Coachs & $38,3 \%$ & $36,7 \%$ & $20,0 \%$ & $5,0 \%$ \\
\hline Coaching-Datenbanken Z.B. Rauen Datenbank, CoachGuide, etc. & $6,1 \%$ & $31,8 \%$ & $40,9 \%$ & $21,2 \%$ \\
\hline Aktive Ansprache durch Coachs & $7,2 \%$ & $36,2 \%$ & $33,3 \%$ & $23,2 \%$ \\
\hline Anzeigen in Fachzeitschriften, Zeitungen, Branchenbüchern, etc. & $0,0 \%$ & $23,9 \%$ & $31,3 \%$ & $44,8 \%$ \\
\hline Fachbücher & $6,0 \%$ & $32,8 \%$ & $31,3 \%$ & $29,9 \%$ \\
\hline TV- und Rundfunkbeiträge & $0,0 \%$ & $4,5 \%$ & $32,8 \%$ & $62,7 \%$ \\
\hline Direktmarketing/Mailing/Newsletter/Flyer & $1,5 \%$ & $6,1 \%$ & $47,0 \%$ & $45,5 \%$ \\
\hline Artikelautorenschaft in wissenschaftlichen Zeitschriften & $7,5 \%$ & $37,3 \%$ & $37,3 \%$ & $17,9 \%$ \\
\hline Artikelautorenschaft in Praktikerzeitschriften & $12,1 \%$ & $31,8 \%$ & $31,8 \%$ & $24,2 \%$ \\
\hline Gezielte Suche bei Berufsverbänden & $8,8 \%$ & $33,8 \%$ & $44,1 \%$ & $13,2 \%$ \\
\hline
\end{tabular}


Abb. 12 Auswahlkriterien für Coaching-Pools

\begin{tabular}{|l|r|r|r|r|}
\hline Auswahlkriterien zur Aufnahme in den Coaching-Pool \\
\hline n=67 & sehr wichtig & \multicolumn{1}{|c|}{ wichtig } & weniger wichtig & unwichtig \\
\hline Referenzen/positive Erfahrungen mit dem Coach durch Dritte & $62,7 \%$ & $31,3 \%$ & $4,5 \%$ & $1,5 \%$ \\
\hline Schwerpunktkompetenzen des Coachs & $50,7 \%$ & $43,3 \%$ & $6,0 \%$ & $0,0 \%$ \\
\hline Ausbildung des Coachs & $50,0 \%$ & $44,1 \%$ & $5,9 \%$ & $0,0 \%$ \\
\hline Verbandszertifizierung & $14,1 \%$ & $35,9 \%$ & $29,7 \%$ & $20,3 \%$ \\
\hline Branchenerfahrung & $20,9 \%$ & $53,7 \%$ & $22,4 \%$ & $3,0 \%$ \\
\hline Kosten/Preis & $13,6 \%$ & $63,6 \%$ & $21,2 \%$ & $1,5 \%$ \\
\hline Ethisches Selbstverständnis des Coachs & $49,3 \%$ & $44,8 \%$ & $4,5 \%$ & $1,5 \%$ \\
\hline Berufserfahrung als Coach & $55,2 \%$ & $41,8 \%$ & $1,5 \%$ & $1,5 \%$ \\
\hline Führungserfahrung & $29,9 \%$ & $47,8 \%$ & $19,4 \%$ & $3,0 \%$ \\
\hline Regionale Nähe & $16,7 \%$ & $45,5 \%$ & $27,3 \%$ & \\
\hline
\end{tabular}

Das wichtigste Kriterium scheinen wieder Referenzen/ positive Erfahrungen mit dem Coach durch Dritte zu sein (62,7\% ,sehr wichtig“; $31,3 \%$,wichtig“). Als ebenfalls bedeutsam erweist sich die Coaching-Erfahrung in Jahren (55,2\% ,sehr wichtig“; $41,8 \%$,wichtig“). Überraschend ist, dass das ethische Selbstverständnis des Coachs (49,3\% „sehr wichtig“; 44,8\% ,wichtig“) den Kunden ebenso wichtig zu sein scheint, wie Schwerpunktkompetenzen $(50,7 \%$ „sehr wichtig“; 43,3\% ,wichtig“) und Ausbildung (50\% „sehr wichtig“; 44,1\%, ,wichtig“).

In der Umfrage aus dem Jahr 2013 wurde ergänzend zur Frage der Anforderungen an Coachs zur Aufnahme in den Coaching-Pool die Frage gestellt, ob Unterschiede bei den Anforderungen an interne und externe Coachs bestehen. Diese Frage wurde 2013 von etwa einem Drittel der Kunden $(35 \%)$ positiv beantwortet, für zwei Drittel bestehen keine Unterschiede in den Anforderungen (65\%) $(n=46)$.

Grundsätzlich wurde angemerkt, dass externe Coachs verstärkt in den höheren Hierarchieebenen eingesetzt werden. Für einen Einsatz im Top Management sind „hochrangige eigene Führungserfahrung" eine wichtige Voraussetzung. Mehrfach wurde ,internationale und eine Branchen übergreifende Qualifikation“ als Anforderung genannt. Ebenfalls wurden „externes Fachwissen“ sowie die ,unabhängige externe Sicht" als wichtige Anforderungen an externe Coachs formuliert.

Als Vorteile für den Einsatz von internen Coachs sehen Kunden deren „Wissensvorsprung für interne Themen“. Eine geringere Coaching-Erfahrung wird durch den internen Wissensvorsprung kompensiert. Als kostengünstigere Alternative werden ,geringere Anforderungen an die Coaching-Ausbildung und -Erfahrung" in Kauf genommen. Vorteilhaft wirkt sich auch eine „leichter überprüfbare Kompetenz" von internen Coachs aus. Jedoch bleibt ebenfalls anzumerken, dass der „Einsatzfokus Interner (häufig) auf Fachthemen oder Begleitung von Projektmanagement beschränkt" bleibt.

\section{Fazit und Ausblick}

Die beschriebenen Veränderungen im Markt bieten Raum für Spekulationen. Ist die Grenze des Wachstums des deutschen Coaching-Marktes langsam erreicht? Aus ökonomischer Sicht spricht ein eintretender Differenzierungswettbewerb, wie er sich bspw. durch neue Formatangebote in Form des Coachings mit neuen Medien andeutet, dafür, dass eine Sättigungsgrenze erreicht sein könnte. Die Coaching-Pools vieler großer Unternehmen sind bereits gefüllt und werden allenfalls noch durch Austausch verändert. Nach wie vor scheinen jedoch noch Entwicklungspotentiale für die Nachfrage bei mittelständischen Unternehmen zu bestehen.

Die weitere Entwicklung des Coaching-Marktes in Bezug auf sein Volumen wird in Zukunft davon abhängen, inwieweit es gelingen kann, zusätzliche Nachfrage zu generieren. Das kann zum einen über eine erhöhte Penetrationsrate gelingen, also bestehenden Kunden mehr Coaching-Dienstleistungen $-z$. B. verstärkt auch für Nachwuchsführungskräfte oder das mittlere Management - zu verkaufen, oder aber zum anderen tatsächlich durch das Gewinnen neuer Kunden. Diese neuen Kundensegmente können im bestehenden Markt durch ein differenziertes Angebot entstehen, also dem Anbieten bspw. neuer Coaching-Formate wie Gesundheits-Coaching als Präventivmaßnahme zum Erhalt der Leistungsfähigkeit am Arbeitsplatz oder dem Erschließen von Kundensegmenten, die bisher Coaching noch nicht in dem Umfang nutzen, wie sie könnten. Marktentwicklungspotential besteht in dieser Hinsicht sicherlich bei mittelständischen Unternehmen.

Insgesamt wird die Entwicklung des Marktes auch davon abhängen, wie sich das Image von Coaching weiter entwickelt. Coaching ist eine wissensintensive und erklärungsbedürftige Dienstleistung. Es ist wichtig, offen und ehrlich über Chancen und Risiken von Coaching zu sprechen. Für die Bewerbung und Imagearbeit steht jede/r Coach in der Verantwortung. Marketing und Imagepflege kann nicht nur durch Verbände betrieben werden. In der Coaching-Studie wurden Coachs und Kunden gebeten, Faktoren zu bewerten, die die Wettbewerbssituation im Markt beeinflussen (vgl. Abb. 13). Das Image spielt im Wettbewerb unter Coachs eine ganz entscheidende Rolle. 39,8\% der Coachs und 33,3\% der Kunden markierten diesen Faktor in seinem Einfluss als „sehr stark“; $54,1 \%$ der Coachs und 45,8\% der Kunden als „stark“. Neben Service-/Kundenorientierung und Kundentreue wurden explizit auch die Anzahl der Wettbewerber 
Abb. 13 Wettbewerbsfaktoren im Coaching-Markt

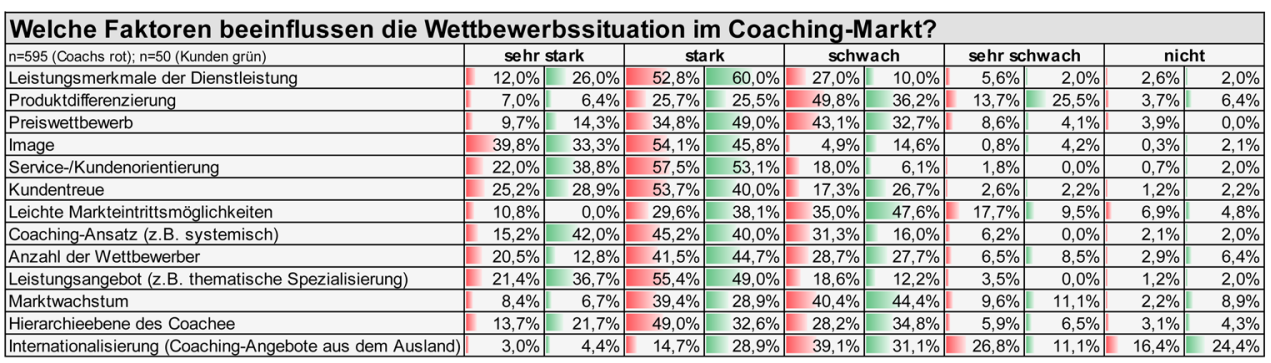

(20,5 und $12,8 \%$ ) sowie das Leistungsangebot, also die thematische Spezialisierung, als stark beeinflussende Faktoren markiert (21,4 und 36,7\%). Die Markierungen zur Spezialisierung decken sich mit Ergebnissen der ersten Marburger Coaching-Studie, wonach Coachs, die sich spezialisieren, signifikant höhere Auftrags- und Umsatzzahlen realisieren konnten, als Coachs, die sich nicht spezialisieren (Gross 2011).

Es wird ein Thema für die nächsten Coaching-Studien sein, die aufgelisteten Wettbewerbsfaktoren genauer zu analysieren. Insgesamt zeichnen die vorliegenden Ergebnisse ein positives Bild für die nähere Zukunft des CoachingMarktes. Auftrags- und Umsatzzahlen sind einigermaßen stabil und es besteht weiterhin Wachstumspotential. Es verdichten sich zudem die Indizien der zunehmenden Professionalisierung des Marktes.

Open Access Dieser Artikel wird unter der Creative Commons Namensnennung 4.0 International Lizenz (http://creativecommons. org/licenses/by/4.0/deed.de) veröffentlicht, welche die uneingeschränkte Nutzung, Verbreitung und Wiedergabe für beliebige Zwecke erlaubt, sofern Sie den/die ursprünglichen Autor(en) und die Quelle ordnungsgemäß nennen, einen Link zur Creative Commons Lizenz beifügen und angeben, ob Änderungen vorgenommen wurden.

\section{Literatur}

Bose, I., \& Neuber, B. (Hrsg.). (2011). Interpersonelle Kommunikation: Analyse undOptimierung. Frankfurt a. M.: Peter Lang.

Charan, R., \& Colvin, G. (1999). Why CEOs fail. Fortune, 139, 68-75.

Dotlich, D. L., \& Cairo, P. C. (1999). Why CEOs fail. San Francisco: Jossey-Bass.

Fengler, J. (2001). Coaching. Definition, Prinzipien, Qualifikationen, illustriert anhand einer Fall-Vignette. Zeitschrift für angewandte Sozialpsychologie - Gruppendynamik und Organisationsberatung, 32(1), 37-60.

Geißler, H. (2008). E-Coaching. Baltmannsweiler: Schneider. Hohengeheren.

Geißler, H., \& Stephan, M. (2014). Das medien-technische Innovationsdefizit von Coaching und eine Möglichkeit, es zu überwinden. In S. M. Weber, M. Göhlich, A. Schröer, \& J. Schwarz (Hrsg.), Organisation und das Neue. Beiträge der Kommission Organisationspädagogik (S. 351-361). Wiesbaden: Springer.
Greco, J. (2001). Hey Coach! Journal of Business Strategy, 22, 28-31. Greif, S. (2008). Coaching und ergebnisorientierte Selbstreflexion. Göttigen: Hogrefe.

Gross, P.-P. (2011). Spezialisierung ist Trumpf - Marketing für externe Dienstleister am Beispiel Coaching. In I. Bose \& B. Neuber (Hrsg.), Interpersonelle Kommunikation: Analyse und Optimierung (S. 345-353). Frankfurt a. M.: Peter Lang.

Gross, P.-P., \& Stephan, M. (2011). Coaching - Der Boom und seine Nebenwirkungen. Zeitschrift für Führung und Organisation (zfo), 81(4), 221-228.

Hogan, R., Curphy, G. J., \& Hogan, J. (1994). What we know about leadership effectiveness and personality. American Psychologist, 49, 493-504.

Höher, P. (2007). Coaching als Methode des Organisationslernens. Bergisch-Gladbach: EHP.

Kelle, U. (2008). Die Integration qualitativer und quantitativer Methoden in der empirischen Sozialforschung (2. Aufl.). Wiesbaden: VS-Springer.

Kühl, S., Strodtholz, P., \& Taffertshofer, A. (2005). Quantitative Methoden der Organisationsforschung. Wiesbaden: VS-Springer.

Looss, W. (1997). Unter vier Augen. Coaching für Manager (4., vollst. überarb. Aufl.). Landsberg am Lech: Moderne Industrie.

Neges, G., \& Neges, R. (2008). Führungskraft und Coaching. Wien: Linde.

Niemes, J. (2002). Discovering the values of executive coaching as a business transformal tool. Journal of Organizational Excellence, $21,61-69$.

Rauen, C. (Hrsg.). (2005). HandbuchCoaching. Göttingen: Hogrefe.

Rauen, C. (2008a). Coaching (2., akt. Aufl.). Göttigen: Hogrefe.

Rauen, C. (2008b). Zwang zur Professionalisierung. Harvard Business Manager, 3, 38-44.

Rückle, H. (2005). Gruppen-Coaching. In C. Rauen (Hrsg.), Handbuch Coaching (S. 183-198). Göttingen: Hogrefe.

Schmid, B. (2005). Coaching und Team-Coaching aus systemischer Perspektive. In C. Rauen (Hrsg.), Handbuch Coaching (S. 109121). Göttingen: Hogrefe.

Stephan, M., Gross, P.-P., \& Hildebrandt, N. (2010). Management von Coaching. Stuttgart: Kohlhammer.

Taffertshofer, A. (2007). Das Coaching der Organisation. Wozu Organisationen Coaching nutzen. Saarbrücken: VDM. 